\title{
BOUND FOR THE ZEROS OF POLYNOMIALS
}

\author{
M. BidKham, A. Zireh And H. A. Soleiman MeZerui
}

Abstract. In this paper, we prove a result concerning the location of the zeros of polynomials in an annulus involving binomial coefficients and $(t, s)$-Fibonacci numbers. Our result includes not only some known results as special cases but also sharpens them more accurate.

Mathematics subject classification (2010): 30C10, 30C15.

Keywords and phrases: Complex polynomial, zeros, $(t, s)$-Fibonacci numbers.

\section{REFERENCES}

[1] C. Affane-Aji, S. Biaz, N. K. Govil, On annuli containing all the zeros of a polynomial, Mathematical and Computer Modeling 52 (2010) 1532-1537.

[2] M. Bidkham, E. Shashahani, An annulus for the zeros of polynomials, Appl. Math. Lett., 24 (2011), 122-125.

[3] J. L. Diaz-Barerro, An annulus for the zeros of polynomials, J. Math. Anal. Appl., 273 (2002), 349-352.

[4] J. L. Diaz-Barerro, J. J. Egozcue, Bounds for the moduli of zeros, Appl. Math. Lett., 17 (2004), 993-996. 